Supporting Information (JA05800385)

\title{
Regiospecific Radical Polymerization of a Tetra-Substituted Ethylene Monomer with Molecular Oxygen for the Synthesis of a New Degradable Polymer
}

\author{
Akikazu Matsumoto* and Shuji Taketani \\ Department of Applied Chemistry, Graduate School of Engineering, Osaka City University, \\ Sugimoto, Sumiyoshi-ku, Osaka 558-8585, Japan \\ E-mail: matsumoto@a-chem.eng.osaka-cu.ac.jp
}

\section{Contents}

Warning for the Treatment of Peroxides

Experimental Procedures (General Methods, Synthesis of Monomers, Polymerization) DFT Calculations

NMR Spectra

TG/TDA Results

Results of Isothermal Degradation

Warning for the Treatment of Peroxides. During all the procedures of isolation and purification, peroxide polymers should be treated without overheating and contact with any reducing agent and metal to avoid explosive decomposition, although it can be handled using the conventional procedures at room temperature. See also the notes for the treatment of low-molecular-weight organic peroxides in a textbook.

\section{Experimental Procedures}

General Methods. The number- and weight-average molecular weights $\left(M_{\mathrm{n}}\right.$ and $\left.M_{\mathrm{w}}\right)$ were determined by gel permeation chromatography (GPC) at $30^{\circ} \mathrm{C}$ in THF using a Tosoh GPC-8000 series system and calibrated with standard polystyrenes. The NMR spectra were recorded on a JEOL JNM A-400 spectrometer. The degradation temperature was determined from the TG/DTA 
results using a SEIKO TG/DTA 6200 at the heating rate of $10^{\circ} \mathrm{C} / \mathrm{min}$ in a nitrogen stream. The sample weight was approximately $1 \mathrm{mg}$. The $T_{95}$ and $T_{50}$ are the 95 and 50 weight $\%$ temperatures, respectively, determined by TG. The initial and maximum degradation temperatures, $T_{\text {init }}$ and $T_{\max }$, respectively, were estimated from the onset and peak temperatures of an exothermic peak in the DTA curve.

Synthesis of Monomer. Ethyl 4,5-dimethyl-2,4-hexadienoate (1): Sodium hydride in oil (1.42 g, $0.036 \mathrm{~mol})$ was dissolved in dry THF $(100 \mathrm{~mL})$ under a nitrogen atmosphere, and triethylphosphonoacetate $(7.1 \mathrm{~mL}, 0.036 \mathrm{~mol})$ was dropwise added with stirring to the solution at room temperature. To the solution, 2,3-dimethyl-2-butenal $(0.036 \mathrm{~mol})$ in a dry THF was then added at $0^{\circ} \mathrm{C}$ over $1 \mathrm{~h}$. After stirring for 1 day, the reaction mixture was filtered, and the solvent was evaporated under reduced pressure. The corresponding ethyl esters were isolated by silica gel column chromatography. The product was again purified by silica gel column chromatography using chloroform. Yield 56\%.

1: liquid, ${ }^{1} \mathrm{H}$ NMR (400 MHz, $\left.\mathrm{CDCl}_{3}\right) \delta 7.88(\mathrm{~d}, J=15.2 \mathrm{~Hz}, \mathrm{C} \underline{\mathrm{H}}=\mathrm{CHCO}, 1 \mathrm{H}), 5.79$ (d, $J=15.2$ $\mathrm{Hz}, \mathrm{C} \underline{\mathrm{H}}=\mathrm{CHCO}, 1 \mathrm{H}), 4.22$ (q, $\left.J=7.2 \mathrm{~Hz}, \mathrm{OC}_{2} \mathrm{CH}_{3}, 2 \mathrm{H}\right), 1.79\left(\mathrm{~s}, \mathrm{CH}_{3}, 3 \mathrm{H}\right), 1.88\left(\mathrm{~s}, \mathrm{CH}_{3}, 3 \mathrm{H}\right)$, $1.97\left(\mathrm{~s}, \mathrm{CH}_{3}, 3 \mathrm{H}\right), 1.31\left(\mathrm{t}, \mathrm{J}=7.2 \mathrm{~Hz}, \mathrm{OCH}_{2} \mathrm{CH}_{3}, 3 \mathrm{H}\right) ;{ }^{13} \mathrm{C}$ NMR (100 MHz, $\left.\mathrm{CDCl}_{3}\right) \delta 168.14$ $(\mathrm{C}=\mathrm{O}), \quad 143.92 \quad(\underline{\mathrm{CH}}=\mathrm{CHCO}), \quad 141.63 \quad\left(\left(\mathrm{CH}_{3}\right)_{2} \mathrm{C}=\underline{\mathrm{CCH}_{3}}\right), \quad 125.87 \quad\left(\left(\mathrm{CH}_{3}\right)_{2} \mathrm{C}=\mathrm{CCH}_{3}\right), \quad 115.42$ $(\mathrm{CH}=\underline{\mathrm{CHCO}}), 60.07\left(\mathrm{O}_{\mathrm{CH}} \mathrm{CH}_{3}\right), 22.52,20.92$, and $14.35\left(\left(\underline{\mathrm{CH}_{3}}\right)_{2} \mathrm{C}=\mathrm{C}^{\mathrm{C}} \mathrm{H}_{3}\right), 14.05\left(\mathrm{OCH}_{2} \underline{\mathrm{C}} \mathrm{H}_{3}\right)$

Polymerization. The polymerization of 1 was carried out in a Pyrex tube with bubbling $\mathrm{O}_{2}$ at $30^{\circ} \mathrm{C}$ for $6 \mathrm{~h}$ in the presence of 2,2'-azobis(4-methoxy-2,4-dimethylvaleronitrile) (Wako Pure Chemical Industries, Ltd., Japan) (monomer/initiator $=50 / 1 \mathrm{w} / \mathrm{w}) .1,2$-Dichloroethane was used as the solvent (monomer/ solvent $=1 / 1 \mathrm{w} / \mathrm{w}$ ). After the polymerization, the contents of the tube were poured into a large amount of $n$-hexane to isolate the resulting polymer. The polymer was dried in vacuo at room temperature, and purified by reprecipitation. Yield $13 \% . M_{\mathrm{n}}=5.6 \times 10^{3}, M_{\mathrm{w}} / M_{\mathrm{n}}=1.4$ (by GPC).

\section{DFT Calculations}

All calculations were carried out using Spartan'04. The values in parentheses are the total energies of the formed allyl radicals (unit in Hartree). Because the produced adduct radicals (peroxy radicals) include two asymmetric carbon centers, leading to a set of diastereomers of the $S, S$ - and $S, R$-forms (or $R, R$ - and $R, S$-), the energies were calculated for each diastereomer. The difference in the values of the two diastereomers was small. We used a smaller value for the discussion. 
Allyl radical from 1 via Path A (-692.227234)

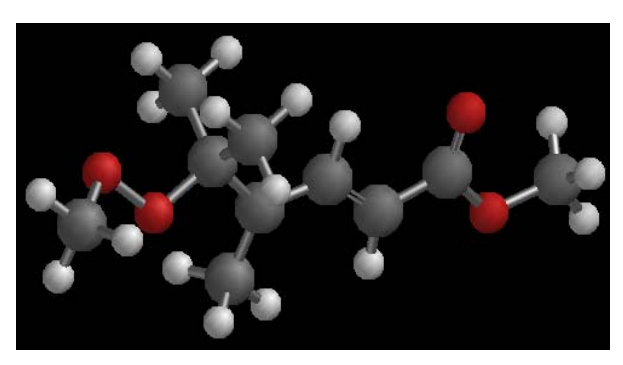

$\begin{array}{rrrrr}\mathrm{C} & 1 & 0.081318 & 0.047536 & -0.05931 \\ \mathrm{C} & 2 & 0.023394 & -0.12071 & 0.084001 \\ \mathrm{C} & 3 & -0.09507 & 0.01036 & 0.009304 \\ \mathrm{H} & 4 & -0.00153 & 0.147916 & -0.11297 \\ \mathrm{C} & 5 & 0.006585 & 0.00985 & -0.07757 \\ \mathrm{H} & 6 & 0.022638 & -0.10611 & 0.147169 \\ \mathrm{C} & 7 & -0.05401 & 0.008465 & -0.00417 \\ \mathrm{H} & 8 & 0.058771 & -0.08124 & -0.13372 \\ \mathrm{H} & 9 & 0.11143 & 0.125265 & 0.030515 \\ \mathrm{H} & 10 & 0.007613 & -0.11037 & 0.137564 \\ \mathrm{C} & 11 & 0.005061 & -0.04933 & 0.041057 \\ \mathrm{H} & 12 & 0.154548 & 0.023272 & -0.07881 \\ \mathrm{H} & 13 & -0.11621 & -0.0215 & -0.12324 \\ \mathrm{H} & 14 & -0.03912 & 0.144987 & 0.077674 \\ \mathrm{C} & 15 & -0.00854 & 0.064807 & -0.03755 \\ \mathrm{H} & 16 & 0.05994 & 0.039797 & 0.153182 \\ \mathrm{H} & 17 & -0.15174 & -0.08001 & 0.0228 \\ \mathrm{H} & 18 & 0.105282 & -0.1209 & -0.06615 \\ \mathrm{C} & 19 & 0.255876 & -0.00703 & 0.165352 \\ \mathrm{O} & 20 & -0.43747 & 0.176424 & -0.22794 \\ \mathrm{O} & 21 & 0.199661 & -0.14546 & 0.087599 \\ \mathrm{C} & 22 & -0.00591 & 0.006101 & 0.000497 \\ \mathrm{H} & 23 & -0.17445 & -0.01983 & 0.049781 \\ \mathrm{H} & 24 & 0.00124 & 0.069087 & -0.16909 \\ \mathrm{H} & 25 & 0.084141 & -0.16652 & 0.000203 \\ \mathrm{O} & 26 & -0.08633 & 0.094619 & 0.134679 \\ \mathrm{O} & 27 & 0.182627 & -0.00445 & -0.05864 \\ \mathrm{C} & 28 & -0.02019 & -0.0113 & 0.018281 \\ \mathrm{H} & 29 & -0.04518 & 0.066567 & 0.158675 \\ \mathrm{H} & 30 & -0.00815 & 0.135587 & -0.12259 \\ \mathrm{H} & 31 & -0.11624 & -0.1259 & -0.0466\end{array}$

Allyl radical from 1 via Path B (-692.209432)

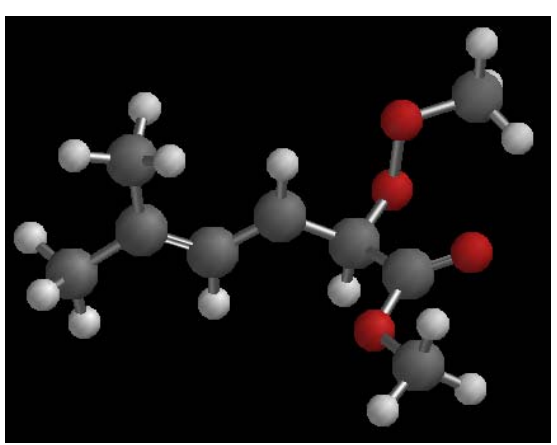

$\begin{array}{rrrrr}\text { H } & 1 & 0.134774 & -0.10512 & -0.0283 \\ \mathrm{C} & 2 & 0.01057 & 0.029762 & 0.077102 \\ \mathrm{H} & 3 & -0.14572 & -0.09709 & -0.01833 \\ \mathrm{H} & 4 & 0.000213 & 0.122492 & -0.12652 \\ \mathrm{C} & 5 & -0.0226 & -0.04393 & 0.000896 \\ \mathrm{C} & 6 & -0.07821 & 0.015019 & -0.01222 \\ \mathrm{C} & 7 & -0.01188 & -0.02643 & 0.003767 \\ \mathrm{H} & 8 & 0.007999 & -0.17988 & 0.007485 \\ \mathrm{C} & 9 & 0.014435 & 0.038407 & 0.00041 \\ \mathrm{H} & 10 & 0.022046 & 0.186384 & -0.00458 \\ \mathrm{C} & 11 & -0.04775 & 0.228693 & -0.01045 \\ \mathrm{O} & 12 & 0.025683 & -0.50752 & 0.016399 \\ \mathrm{O} & 13 & 0.058462 & 0.256388 & -0.00304 \\ \mathrm{C} & 14 & -0.00215 & -0.00612 & 1.14 \mathrm{E}-05 \\ \mathrm{H} & 15 & 0.006756 & -0.10313 & 0.149172 \\ \mathrm{H} & 15 & 0.006756 & -0.10313 & 0.149172 \\ \mathrm{H} & 16 & 0.029718 & -0.1092 & -0.14183 \\ \mathrm{H} & 17 & 0.133246 & 0.128876 & 0.007848 \\ \mathrm{O} & 18 & -0.05889 & 0.150007 & 0.025592 \\ \mathrm{O} & 19 & 0.055964 & -0.10609 & -0.15536 \\ \mathrm{C} & 20 & 0.007551 & 0.016611 & 0.02113 \\ \mathrm{H} & 21 & -0.02722 & 0.176905 & -0.01184 \\ \mathrm{H} & 22 & -0.1606 & -0.08511 & -0.02725 \\ \mathrm{H} & 23 & 0.058035 & -0.03924 & 0.162772 \\ \mathrm{C} & 24 & 0.012994 & 0.046654 & -0.06228 \\ \mathrm{H} & 25 & -0.14997 & -0.09021 & -0.0055 \\ \mathrm{H} & 26 & 0.126313 & -0.11906 & -0.01295 \\ \mathrm{H} & 27 & 0.014369 & 0.075239 & 0.157685 \\ \mathrm{C} & 28 & 0.020154 & -0.0497 & 0.004357 \\ \mathrm{H} & 29 & -0.08962 & 0.043439 & -0.14324 \\ \mathrm{H} & 30 & -0.09332 & 0.061269 & 0.132966 \\ \mathrm{H} & 31 & 0.148638 & 0.091682 & -0.00389 \\ & & & & \end{array}$


Allyl radical from 2 via Path A (-652.905311)

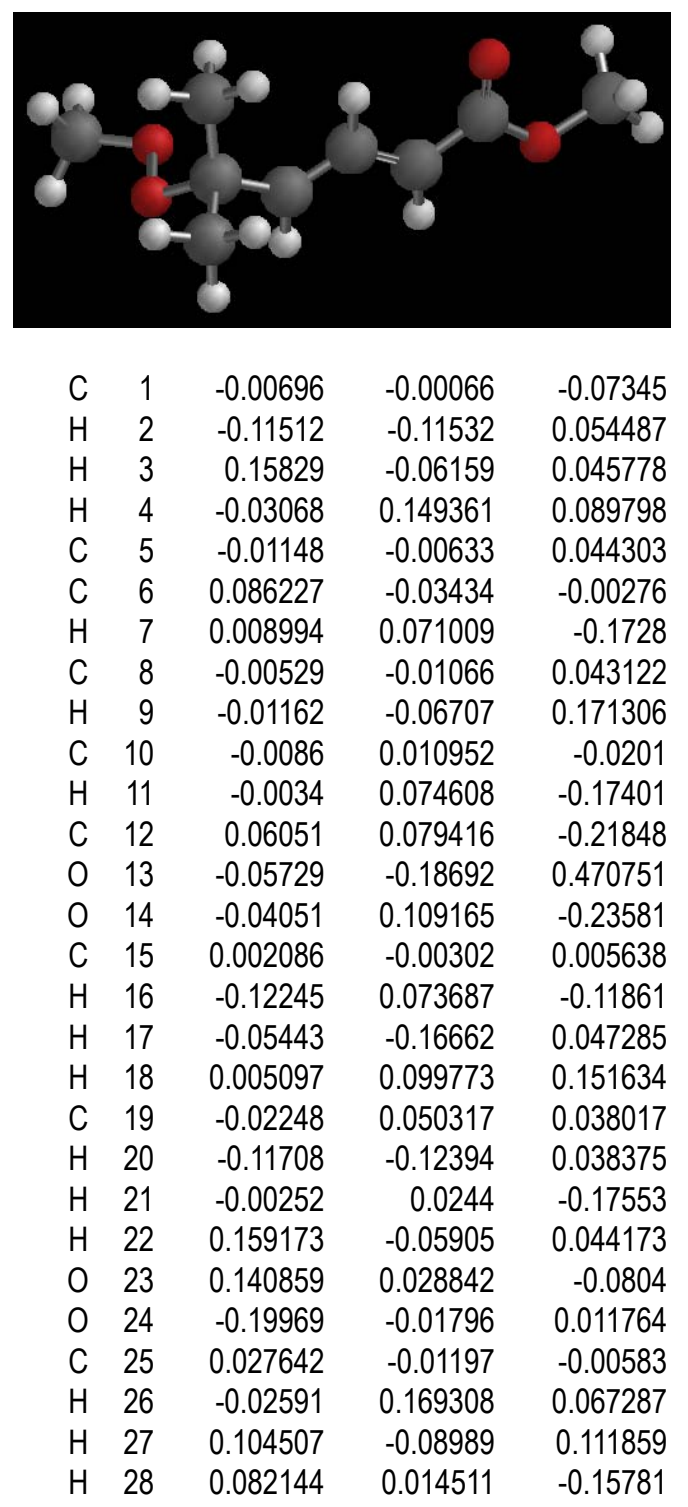

Allyl radical from 2 via Path B (-652.892354)

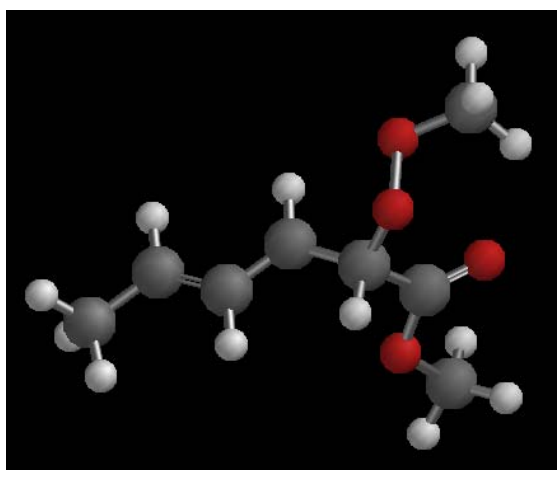

\begin{tabular}{|c|c|c|c|}
\hline C 1 & -0.03974 & -0.01277 & -0.0168 \\
\hline $\mathrm{H} 2$ & 0.101406 & -0.12726 & 0.046677 \\
\hline $\mathrm{H} 3$ & 0.090256 & 0.094178 & -0.10745 \\
\hline $\mathrm{H} \quad 4$ & -0.03223 & 0.097684 & 0.142252 \\
\hline C 5 & 0.109154 & -0.03731 & -0.05842 \\
\hline C 6 & -0.00055 & 0.042173 & 0.046507 \\
\hline H 7 & -0.00264 & 0.106173 & 0.14682 \\
\hline C 8 & -0.09316 & -0.011 & -0.00768 \\
\hline H 9 & -0.00595 & -0.11102 & -0.15104 \\
\hline C 10 & -0.00072 & -0.00456 & -0.0726 \\
\hline H 11 & 0.031629 & 0.089769 & 0.154116 \\
\hline C 12 & 0.271472 & -0.04605 & 0.126414 \\
\hline 013 & -0.4917 & -0.08403 & -0.16262 \\
\hline 014 & 0.238345 & 0.103734 & 0.05492 \\
\hline C 15 & -0.00678 & -0.00498 & 0.001358 \\
\hline H 16 & -0.15494 & 0.055508 & 0.078395 \\
\hline H 17 & 0.116662 & 0.144881 & -0.01775 \\
\hline H 18 & -0.04273 & -0.06487 & -0.16519 \\
\hline 019 & -0.09367 & -0.10306 & 0.125591 \\
\hline 020 & 0.182206 & 0.001743 & -0.06537 \\
\hline C 21 & -0.01869 & 0.009227 & 0.020047 \\
\hline H 22 & -0.10885 & 0.138632 & -0.02255 \\
\hline H 23 & -0.02214 & -0.11545 & -0.13997 \\
\hline H 24 & -0.04387 & -0.0859 & 0.149697 \\
\hline C 25 & -0.00486 & 0.036321 & 0.049148 \\
\hline H 26 & 0.166112 & -0.00676 & -0.05517 \\
\hline H 27 & -0.04945 & -0.15947 & 0.032163 \\
\hline H 28 & -0.09457 & 0.054455 & -0.13148 \\
\hline
\end{tabular}


Allyl radical from 3 via Path A (-613.580832)

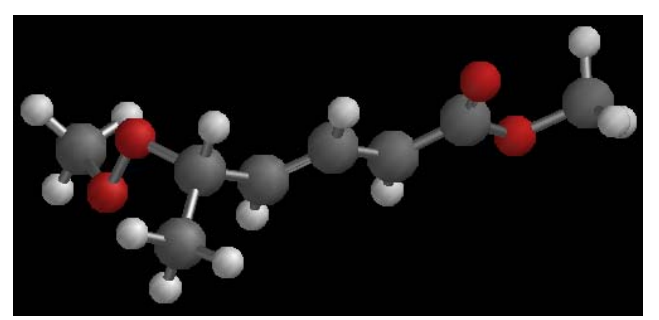

$\begin{array}{rrrrr}\mathrm{C} & 1 & -0.03489 & 0.044473 & 0.034556 \\ \mathrm{H} & 2 & 0.140828 & -0.07235 & 0.077731 \\ \mathrm{H} & 3 & -0.11331 & -0.12567 & -0.03317 \\ \mathrm{H} & 4 & 0.050961 & 0.067461 & -0.15591 \\ \mathrm{C} & 5 & 0.011475 & -0.01032 & -0.08289 \\ \mathrm{H} & 6 & -0.04994 & -0.08013 & 0.158173 \\ \mathrm{C} & 7 & 0.089345 & -0.02555 & 0.014179 \\ \mathrm{C} & 8 & -0.01361 & -0.02348 & 0.029639 \\ \mathrm{H} & 9 & 0.031958 & 0.116792 & -0.14335 \\ \mathrm{H} & 10 & -0.03181 & -0.11294 & 0.142738 \\ \mathrm{O} & 11 & 0.098224 & 0.083789 & 0.119258 \\ \mathrm{O} & 12 & -0.00853 & -0.1587 & -0.11821 \\ \mathrm{C} & 13 & -0.00695 & 0.011188 & 0.024439 \\ \mathrm{H} & 14 & 0.075421 & 0.083242 & -0.14544 \\ \mathrm{H} & 15 & -0.14726 & 0.088288 & 0.046075 \\ \mathrm{H} & 16 & 0.117299 & 0.006879 & 0.135923 \\ \mathrm{C} & 17 & -0.0095 & 0.017524 & -0.01954 \\ \mathrm{H} & 18 & 0.018118 & 0.118672 & -0.14656 \\ \mathrm{C} & 19 & 0.090075 & 0.138383 & -0.17826 \\ \mathrm{O} & 20 & -0.11547 & -0.31053 & 0.389606 \\ \mathrm{O} & 21 & -0.01186 & 0.16813 & -0.20211 \\ \mathrm{C} & 22 & 0.001894 & -0.00419 & 0.004876 \\ \mathrm{H} & 23 & -0.02553 & 0.04746 & 0.173673 \\ \mathrm{H} & 24 & -0.1068 & 0.105583 & -0.10947 \\ \mathrm{H} & 25 & -0.05013 & -0.174 & -0.01596\end{array}$

Allyl radical from 3 via Path B $(-613.566353)$

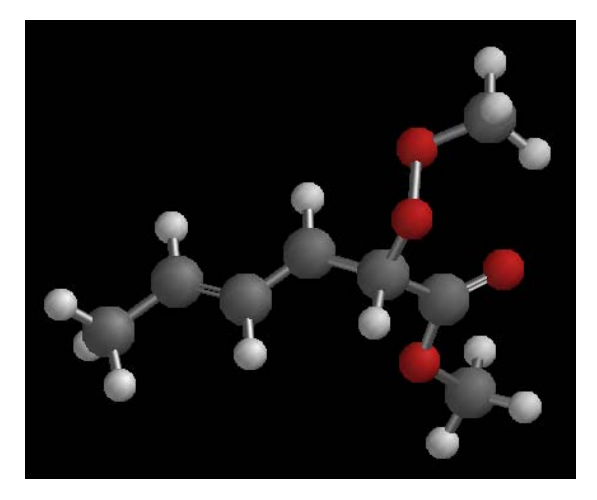

$\begin{array}{rrrrr}\mathrm{C} & 1 & -0.03752 & -0.00625 & 0.014262 \\ \mathrm{H} & 2 & 0.094246 & 0.075837 & 0.120924 \\ \mathrm{H} & 3 & 0.074986 & -0.15082 & -0.02939 \\ \mathrm{H} & 4 & 0.014733 & 0.09571 & -0.14595 \\ \mathrm{C} & 5 & 0.101655 & -0.01777 & 0.016827 \\ \mathrm{H} & 6 & -0.0313 & -0.09585 & 0.154431 \\ \mathrm{C} & 7 & 0.002413 & 0.032436 & -0.04445 \\ \mathrm{H} & 8 & 0.034636 & 0.093482 & -0.1525 \\ \mathrm{C} & 9 & -0.08328 & 0.01137 & -0.01373 \\ \mathrm{H} & 10 & -0.03821 & -0.09631 & 0.157402 \\ \mathrm{C} & 11 & -0.00872 & 0.010717 & 0.074582 \\ \mathrm{H} & 12 & 0.057481 & 0.051066 & -0.16428 \\ \mathrm{C} & 13 & 0.249148 & -0.12901 & -0.11804 \\ \mathrm{O} & 14 & -0.48805 & 0.057743 & 0.183829 \\ \mathrm{O} & 15 & 0.250903 & 0.036577 & -0.07937 \\ \mathrm{C} & 16 & -0.0078 & -0.00371 & -0.00028 \\ \mathrm{H} & 17 & -0.14376 & 0.071369 & -0.08649 \\ \mathrm{H} & 18 & -0.04608 & -0.01948 & 0.175844 \\ \mathrm{H} & 19 & 0.145625 & 0.116309 & -0.01411 \\ \mathrm{O} & 20 & -0.11613 & -0.09558 & -0.11391 \\ \mathrm{O} & 21 & 0.17561 & -0.03604 & 0.070988 \\ \mathrm{C} & 22 & -0.01549 & 0.011226 & -0.02219 \\ \mathrm{H} & 23 & -0.05105 & -0.08478 & 0.153883 \\ \mathrm{H} & 24 & -0.06876 & 0.163874 & -0.00032 \\ \mathrm{H} & 25 & -0.0653 & -0.09212 & -0.13793\end{array}$


Peroxy radical from 1 [5,2-addition, $(S, S)]$ (-842.599915)

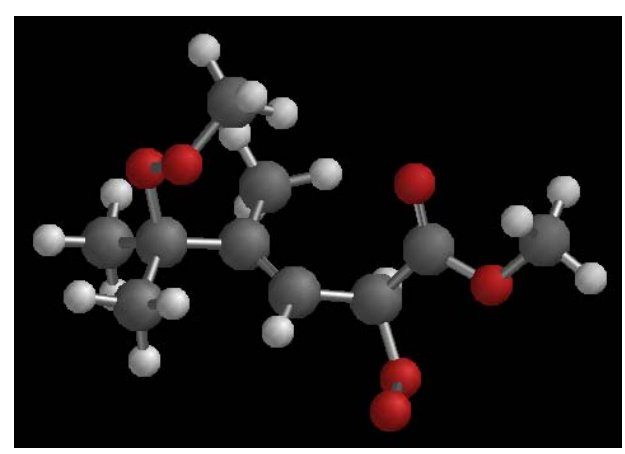

$\begin{array}{rrrrr}\text { H } & 1 & 0.014814 & 0.170936 & -0.01918 \\ \mathrm{C} & 2 & 0.061329 & -0.01995 & 0.033111 \\ \mathrm{H} & 3 & -0.01118 & -0.07152 & -0.16105 \\ \mathrm{H} & 4 & -0.15351 & -0.0325 & 0.082729 \\ \mathrm{C} & 5 & 0.042672 & 0.044344 & 0.032974 \\ \mathrm{C} & 6 & -0.17198 & -0.08383 & -0.11108 \\ \mathrm{C} & 7 & 0.141495 & 0.048171 & 0.030798 \\ \mathrm{H} & 8 & -0.01594 & 0.040052 & 0.182073 \\ \mathrm{C} & 9 & 0.00556 & -0.01526 & 0.07527 \\ \mathrm{H} & 10 & -0.01519 & 0.038304 & -0.18257 \\ \mathrm{C} & 11 & -0.06206 & 0.266106 & 0.043022 \\ \mathrm{O} & 12 & -0.12731 & -0.47755 & -0.1178 \\ \mathrm{O} & 13 & 0.151931 & 0.227578 & 0.068539 \\ \mathrm{C} & 14 & -0.00725 & -0.00502 & -0.0042 \\ \mathrm{H} & 15 & 0.025654 & -0.08648 & -0.15941 \\ \mathrm{H} & 16 & 0.160462 & 0.08318 & 0.054117 \\ \mathrm{H} & 17 & -0.04979 & -0.12599 & 0.124422 \\ \mathrm{O} & 18 & -0.0825 & -0.11246 & -0.05921 \\ \mathrm{O} & 19 & -0.05894 & 0.094701 & 0.15292 \\ \mathrm{C} & 20 & -0.0039 & 0.00346 & -0.03089 \\ \mathrm{H} & 21 & -0.10277 & -0.11233 & -0.09325 \\ \mathrm{H} & 22 & 0.116213 & 0.073798 & -0.1049 \\ \mathrm{H} & 23 & 0.088348 & -0.09728 & 0.127823 \\ \mathrm{O} & 24 & 0.043827 & 0.138129 & -0.1399 \\ \mathrm{O} & 25 & 0.021659 & 0.00129 & 0.190027 \\ \mathrm{C} & 26 & 0.000443 & -0.02084 & -0.07289 \\ \mathrm{H} & 27 & 0.015577 & 0.169719 & 0.006443 \\ \mathrm{H} & 28 & -0.15734 & -0.04304 & 0.068795 \\ \mathrm{H} & 29 & 0.123548 & -0.06709 & 0.107293 \\ \mathrm{C} & 30 & 0.00381 & 0.032379 & 0.064235 \\ \mathrm{H} & 31 & 0.160464 & -0.0349 & -0.05876 \\ \mathrm{H} & 32 & -0.09142 & -0.1448 & -0.02805 \\ \mathrm{H} & 33 & -0.06673 & 0.118694 & -0.10146\end{array}$

Peroxy radical from 1 [5,4-addition, $(S, S)]$ $(-842.609870)$

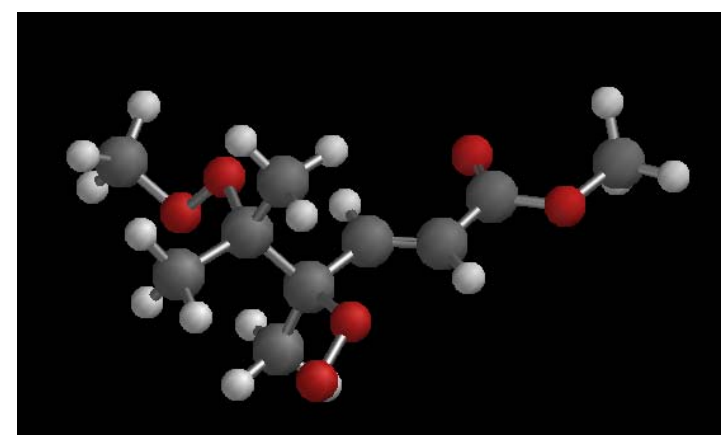

$\begin{array}{lllll}\text { C } & 1 & -0.0672 & -0.02696 & -0.01552\end{array}$

$\begin{array}{lllll}\mathrm{H} & 2 & 0.07623 & -0.03861 & 0.154765\end{array}$

$\begin{array}{lllll}\mathrm{H} & 3 & 0.110686 & -0.04854 & -0.12659\end{array}$

$\begin{array}{lllll}\mathrm{H} & 4 & -0.01097 & 0.17141 & -0.00842\end{array}$

$\begin{array}{lllll}\text { C } & 5 & -0.01405 & 0.043893 & 0.06095\end{array}$

$\begin{array}{lllll}\text { C } & 6 & 0.084896 & 0.021326 & -0.02803\end{array}$

$\begin{array}{lllll}\text { C } & 7 & 0.124266 & 0.000357 & 0.018753\end{array}$

$\begin{array}{lllll}\mathrm{H} & 8 & 0.030908 & -0.18321 & 0.031173\end{array}$

$\begin{array}{lllll}\text { C } & 9 & -0.06597 & 0.030972 & -0.01385\end{array}$

$\begin{array}{lllll}\mathrm{H} & 10 & -0.05051 & 0.182043 & -0.03279\end{array}$

$\begin{array}{lllll}\text { C } & 11 & 0.028832 & 0.260264 & -0.03574\end{array}$

$\begin{array}{lllll}\mathrm{O} & 12 & 0.054816 & -0.51166 & 0.08506\end{array}$

$\begin{array}{lllll}0 & 13 & -0.10231 & 0.241449 & -0.05014\end{array}$

$\begin{array}{lllll}\text { C } & 14 & 0.004032 & -0.00411 & 0.001273\end{array}$

$\begin{array}{lllll}\mathrm{H} & 15 & -0.1493 & 0.104148 & -0.03995\end{array}$

$\begin{array}{lllll}H & 16 & 0.018983 & -0.13032 & -0.12592\end{array}$

$\begin{array}{lllll}\text { H } & 17 & -0.02455 & -0.08306 & 0.160257\end{array}$

$\begin{array}{lllll}\mathrm{O} & 18 & -0.01501 & -0.14661 & -0.05944\end{array}$

$\begin{array}{lllll}0 & 19 & -0.09662 & 0.128396 & 0.106299\end{array}$

$\begin{array}{lllll}\text { C } & 20 & 0.006048 & -0.00652 & -0.02606\end{array}$

$\begin{array}{lllll}\mathrm{H} & 21 & 0.134403 & 0.054985 & -0.10316\end{array}$

$\begin{array}{lllll}\mathrm{H} & 22 & 0.068917 & -0.05728 & 0.160843\end{array}$

$\begin{array}{lllll}\mathrm{H} & 23 & -0.08275 & -0.13987 & -0.0792\end{array}$

$\begin{array}{lllll}0 & 24 & -0.15982 & -0.03723 & -0.09569\end{array}$

$\begin{array}{lllll}0 & 25 & 0.103966 & 0.151698 & 0.051509\end{array}$

$\begin{array}{lllll}\text { C } & 26 & 0.018605 & -0.00021 & 0.073642\end{array}$

$\begin{array}{lllll}\mathrm{H} & 27 & -0.15586 & -0.0811 & -0.02799\end{array}$

$\begin{array}{lllll}\mathrm{H} & 28 & -0.01969 & 0.168907 & -0.04426\end{array}$

$\begin{array}{lllll}\mathrm{H} & 29 & 0.116885 & -0.06942 & -0.11331\end{array}$

$\begin{array}{lllll}\text { C } & 30 & -0.0211 & -0.01273 & -0.06466\end{array}$

$\begin{array}{lllll}\mathrm{H} & 31 & 0.044858 & -0.15493 & 0.068114\end{array}$

$\begin{array}{lllll}\mathrm{H} & 32 & 0.140151 & 0.109317 & 0.019237\end{array}$

$\begin{array}{lllll}\mathrm{H} & 33 & -0.13176 & 0.063195 & 0.098846\end{array}$ 




$\begin{array}{rrrrr}\mathrm{C} & 1 & -0.0482 & 0.020253 & 0.037018 \\ \mathrm{H} & 2 & 0.162643 & 0.045837 & 0.052769 \\ \mathrm{H} & 3 & -0.01479 & -0.17237 & 0.013029 \\ \mathrm{H} & 4 & -0.01485 & 0.042122 & -0.17004 \\ \mathrm{C} & 5 & -0.02023 & -0.02533 & 0.047922 \\ \mathrm{C} & 6 & 0.155317 & 0.033907 & -0.04859 \\ \mathrm{H} & 7 & -0.01399 & 0.024553 & -0.18096 \\ \mathrm{C} & 8 & -0.1319 & -0.03416 & 0.041559 \\ \mathrm{H} & 9 & 0.008761 & -0.02352 & 0.188377 \\ \mathrm{C} & 10 & -0.01116 & -0.0028 & 0.065439 \\ \mathrm{H} & 11 & 0.013697 & 0.003263 & -0.1856 \\ \mathrm{C} & 12 & 0.05689 & -0.26818 & -0.08455 \\ \mathrm{O} & 13 & 0.120374 & 0.479116 & 0.172891 \\ \mathrm{O} & 14 & -0.13899 & -0.21957 & -0.097 \\ \mathrm{O} & 15 & 0.087145 & 0.105263 & -0.07789 \\ \mathrm{O} & 16 & 0.057284 & -0.07631 & 0.167815 \\ \mathrm{C} & 17 & 0.005093 & -0.00344 & -0.03291 \\ \mathrm{H} & 18 & -0.09805 & 0.108544 & 0.113034 \\ \mathrm{H} & 19 & 0.106091 & 0.102621 & -0.09914 \\ \mathrm{H} & 20 & -0.11219 & -0.08548 & -0.10458 \\ \mathrm{C} & 21 & -0.00618 & 0.011457 & -0.0723 \\ \mathrm{H} & 22 & -0.11111 & 0.089727 & 0.104301 \\ \mathrm{H} & 23 & -0.02595 & -0.16591 & 0.030548 \\ \mathrm{H} & 24 & 0.164777 & 0.039401 & 0.051448 \\ \mathrm{O} & 25 & -0.01229 & -0.11461 & -0.16016 \\ \mathrm{O} & 26 & -0.03892 & -0.03055 & 0.18196 \\ \mathrm{C} & 27 & 0.00669 & 0.005694 & -0.00056 \\ \mathrm{H} & 28 & 0.007282 & 0.043078 & 0.17882 \\ \mathrm{H} & 29 & 0.008566 & 0.156123 & -0.09503 \\ \mathrm{H} & 30 & -0.1618 & -0.08872 & -0.03761\end{array}$

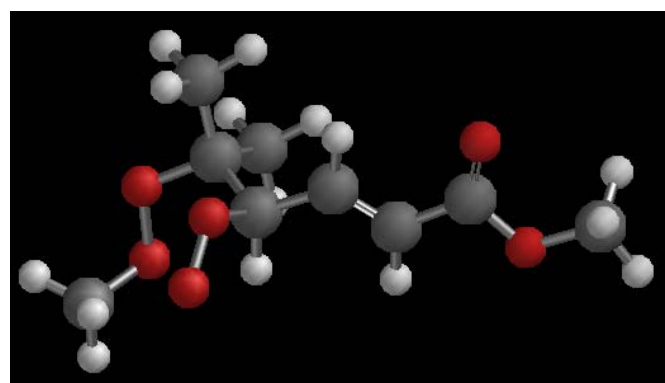

Peroxy radical from 2(5,4-addition) (-803.290644)

$\begin{array}{rrrrr}\mathrm{C} & 1 & 0.018949 & 0.057639 & -0.03273 \\ \mathrm{H} & 2 & -0.11709 & -0.10688 & -0.08083 \\ \mathrm{H} & 3 & 0.15171 & -0.08579 & 0.003632 \\ \mathrm{H} & 4 & -0.0546 & 0.021276 & 0.167628 \\ \mathrm{C} & 5 & 0.061544 & 0.016443 & -0.01472 \\ \mathrm{C} & 6 & -0.01539 & -0.0785 & -0.0006 \\ \mathrm{H} & 7 & -0.01725 & 0.16425 & -0.07698 \\ \mathrm{C} & 8 & -0.13514 & -0.03059 & 0.035244 \\ \mathrm{H} & 9 & 0.043555 & -0.15047 & 0.104721 \\ \mathrm{C} & 10 & 0.064881 & 0.045108 & -0.04317 \\ \mathrm{H} & 11 & -0.02946 & 0.157089 & -0.10452 \\ \mathrm{O} & 12 & -0.16132 & -0.01067 & 0.042273 \\ \mathrm{O} & 13 & 0.128436 & -0.04797 & -0.1358 \\ \mathrm{C} & 14 & -0.00996 & -0.0077 & 0.022185 \\ \mathrm{H} & 15 & 0.048761 & 0.040901 & 0.164086 \\ \mathrm{H} & 16 & -0.01544 & 0.155543 & -0.09687 \\ \mathrm{H} & 17 & -0.16122 & -0.07991 & 0.004693 \\ \mathrm{O} & 18 & -0.00112 & -0.18247 & 0.07857 \\ \mathrm{O} & 19 & -0.04328 & 0.186991 & 0.049673 \\ \mathrm{C} & 20 & -0.12894 & 0.193592 & -0.11488 \\ \mathrm{O} & 21 & 0.153421 & -0.42377 & 0.26464 \\ \mathrm{O} & 22 & -0.00138 & 0.22354 & -0.14891 \\ \mathrm{C} & 23 & -0.00298 & -0.00507 & 0.003119 \\ \mathrm{H} & 24 & 0.037462 & -0.16304 & -0.07312 \\ \mathrm{H} & 25 & 0.094709 & 0.132909 & -0.0912 \\ \mathrm{H} & 26 & 0.053992 & -0.00497 & 0.174678 \\ \mathrm{C} & 27 & -0.01506 & 0.010479 & 0.0833 \\ \mathrm{H} & 28 & -0.11348 & -0.10617 & -0.08533 \\ \mathrm{H} & 29 & 0.009561 & 0.151669 & -0.0909 \\ \mathrm{H} & 30 & 0.156111 & -0.07346 & -0.00788 \\ & & & & \end{array}$


Peroxy radical from 3 [5,2-addition, $(S, S)]$ (-763.956698)



$\begin{array}{rrrrr}\text { H } & 1 & 0.019123 & 0.170529 & 0.027397 \\ \mathrm{C} & 2 & 0.044984 & -0.01994 & 0.017991 \\ \mathrm{H} & 3 & -0.01315 & -0.02474 & -0.17229 \\ \mathrm{H} & 4 & -0.1536 & -0.05165 & 0.075342 \\ \mathrm{C} & 5 & 0.015148 & 0.019092 & -0.03113 \\ \mathrm{H} & 6 & 0.003827 & 0.019644 & 0.177368 \\ \mathrm{C} & 7 & -0.15487 & -0.01249 & -0.03669 \\ \mathrm{H} & 8 & 0.000287 & 0.022736 & -0.18303 \\ \mathrm{O} & 9 & -0.09042 & -0.1186 & -0.08485 \\ \mathrm{O} & 10 & -0.05691 & 0.077535 & 0.167887 \\ \mathrm{C} & 11 & -0.00597 & 0.003098 & -0.03104 \\ \mathrm{H} & 12 & -0.10582 & -0.10473 & -0.0972 \\ \mathrm{H} & 13 & 0.111814 & 0.085176 & -0.10599 \\ \mathrm{H} & 14 & 0.098365 & -0.10604 & 0.115094 \\ \mathrm{C} & 15 & 0.137994 & 0.020636 & 0.031267 \\ \mathrm{H} & 16 & -0.00034 & -0.02714 & 0.18918 \\ \mathrm{C} & 17 & 0.014505 & -0.0136 & 0.06302 \\ \mathrm{H} & 18 & -0.02098 & 0.036827 & -0.18129 \\ \mathrm{C} & 19 & -0.05873 & 0.276746 & -0.0416 \\ \mathrm{O} & 20 & -0.11083 & -0.49644 & 0.115693 \\ \mathrm{O} & 21 & 0.132037 & 0.229816 & -0.08016 \\ \mathrm{C} & 22 & -0.0063 & -0.00562 & -0.00101 \\ \mathrm{H} & 23 & -0.02049 & -0.1411 & -0.11444 \\ \mathrm{H} & 24 & 0.15879 & 0.092335 & -0.04071 \\ \mathrm{H} & 25 & 0.009346 & -0.06776 & 0.171247 \\ \mathrm{O} & 26 & -0.00098 & 0.147345 & -0.13119 \\ \mathrm{O} & 27 & 0.053179 & -0.01167 & 0.181122 \\ & & & & \end{array}$

Peroxy radical from 3 [5,4-addition, $(S, R)]$ (-763.965825)



$\begin{array}{rrrrr}\mathrm{C} & 1 & -0.00714 & 0.051203 & -0.00655 \\ \mathrm{H} & 2 & 0.082245 & -0.08079 & -0.13349 \\ \mathrm{H} & 3 & 0.082534 & -0.04652 & 0.150401 \\ \mathrm{H} & 4 & -0.16429 & -0.0647 & 0.008178 \\ \mathrm{C} & 5 & -0.04476 & -0.00876 & -0.0712 \\ \mathrm{H} & 6 & -0.07867 & 0.072312 & 0.137982 \\ \mathrm{C} & 7 & 0.004456 & -0.09451 & 0.031935 \\ \mathrm{H} & 8 & 0.023351 & 0.183241 & -0.00297 \\ \mathrm{C} & 9 & 0.113539 & -0.03166 & -0.03307 \\ \mathrm{H} & 10 & -0.05172 & -0.16285 & -0.07957 \\ \mathrm{C} & 11 & -0.05513 & 0.048211 & 0.034656 \\ \mathrm{H} & 12 & 0.0373 & 0.16791 & 0.080865 \\ \mathrm{C} & 13 & 0.140306 & 0.203464 & 0.085652 \\ \mathrm{O} & 14 & -0.1797 & -0.44818 & -0.20405 \\ \mathrm{O} & 15 & 0.016614 & 0.238708 & 0.119189 \\ \mathrm{C} & 16 & 0.002637 & -0.00515 & -0.00232 \\ \mathrm{H} & 17 & -0.03743 & -0.15487 & 0.089469 \\ \mathrm{H} & 18 & -0.08582 & 0.143121 & 0.083707 \\ \mathrm{H} & 19 & -0.06395 & -0.01884 & -0.17001 \\ \mathrm{O} & 20 & 0.182448 & -0.0317 & -0.00324 \\ \mathrm{O} & 21 & -0.1542 & 0.01896 & 0.117317 \\ \mathrm{C} & 22 & 0.011518 & -0.01518 & -0.01378 \\ \mathrm{H} & 23 & 0.152204 & -0.07478 & 0.062536 \\ \mathrm{H} & 24 & -0.01588 & -0.03662 & -0.17413 \\ \mathrm{H} & 25 & 0.011912 & 0.182549 & 0.021358 \\ \mathrm{O} & 26 & 0.110943 & 0.142425 & -0.07009 \\ \mathrm{O} & 27 & -0.0333 & -0.17699 & -0.05877\end{array}$


Peroxy radical from 4 [2,3-addition, $(S, S)]$ (-575.351100)



$\begin{array}{rrrrr}\mathrm{C} & 1 & 0.037036 & -0.00035 & -0.01695 \\ \mathrm{H} & 2 & -0.10945 & -0.10649 & -0.08247 \\ \mathrm{H} & 3 & 0.003884 & -0.0478 & 0.170051 \\ \mathrm{H} & 4 & -0.07075 & 0.157547 & -0.00916 \\ \mathrm{C} & 5 & -0.14687 & 0.030794 & -0.02951 \\ \mathrm{H} & 6 & 0.006858 & 0.044382 & -0.17868 \\ \mathrm{C} & 7 & 0.142883 & -0.02315 & 0.030392 \\ \mathrm{H} & 8 & -0.00623 & -0.04749 & 0.180869 \\ \mathrm{C} & 9 & 0.011493 & 0.000984 & 0.075527 \\ \mathrm{H} & 10 & -0.01626 & 0.030973 & -0.18272 \\ \mathrm{C} & 11 & -0.04254 & 0.04459 & -0.01567 \\ \mathrm{H} & 12 & 0.050842 & -0.05802 & 0.157962 \\ \mathrm{C} & 13 & 0.008828 & -0.06367 & -0.00203 \\ \mathrm{H} & 14 & -0.12808 & 0.015839 & 0.115038 \\ \mathrm{H} & 15 & 0.131594 & 0.106834 & 0.053676 \\ \mathrm{H} & 16 & -0.05769 & 0.060615 & -0.15327 \\ \mathrm{O} & 17 & 0.064659 & -0.04232 & -0.14594 \\ \mathrm{O} & 18 & -0.05418 & 0.179805 & 0.01255 \\ \mathrm{O} & 19 & 0.011954 & -0.06616 & -0.18053 \\ \mathrm{O} & 20 & 0.039318 & -0.06669 & 0.176004 \\ \mathrm{C} & 21 & -0.01012 & -0.0214 & -0.00426 \\ \mathrm{H} & 22 & 0.05218 & -0.07099 & -0.15742 \\ \mathrm{H} & 23 & 0.146712 & 0.070766 & 0.083825 \\ \mathrm{H} & 24 & -0.06607 & -0.1286 & 0.102709\end{array}$

Peroxy radical from 4 [2,5-addition, $(S, R)]$ $(-575.354876)$

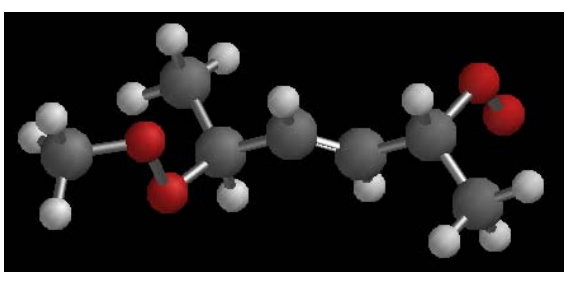

$\begin{array}{rrrrr}\mathrm{C} & 1 & -0.04267 & -0.03094 & 0.030216 \\ \mathrm{H} & 2 & 0.137662 & 0.065148 & 0.090808 \\ \mathrm{H} & 3 & 0.063148 & -0.09257 & -0.1372 \\ \mathrm{H} & 4 & -0.09011 & 0.13443 & -0.06534 \\ \mathrm{C} & 5 & 0.038629 & -0.01892 & -0.05313 \\ \mathrm{H} & 6 & -0.05381 & 0.094473 & 0.150282 \\ \mathrm{C} & 7 & 0.14497 & 0.00687 & -0.01071 \\ \mathrm{H} & 8 & 0.036319 & -0.10502 & -0.14932 \\ \mathrm{C} & 9 & -0.14604 & -0.00671 & 0.004062 \\ \mathrm{H} & 10 & -0.03501 & 0.105568 & 0.149742 \\ \mathrm{C} & 11 & -0.01768 & -0.01821 & 0.078595 \\ \mathrm{H} & 12 & 0.052193 & -0.07058 & -0.1613 \\ \mathrm{C} & 13 & 0.036918 & 0.044382 & -0.03128 \\ \mathrm{H} & 14 & 0.108105 & -0.13302 & 0.026926 \\ \mathrm{H} & 15 & -0.05197 & 0.05743 & 0.158253 \\ \mathrm{H} & 16 & -0.13982 & -0.06132 & -0.08541 \\ \mathrm{O} & 17 & -0.09 & 0.094283 & -0.11681 \\ \mathrm{O} & 18 & 0.169702 & 9.12 \mathrm{E}-05 & 0.097752 \\ \mathrm{C} & 19 & -0.01444 & -0.01022 & -0.02271 \\ \mathrm{H} & 20 & -0.09257 & -0.15012 & -0.01882 \\ \mathrm{H} & 21 & -0.06334 & 0.089881 & 0.146991 \\ \mathrm{H} & 22 & -0.02563 & 0.103591 & -0.14449 \\ \mathrm{O} & 23 & -0.00388 & 0.019564 & 0.188617 \\ \mathrm{O} & 24 & 0.079334 & -0.11809 & -0.12572\end{array}$


Table S-1 Total Energy (E) for Peroxy and Allyl Radicals and the Estimated BDE Values

\begin{tabular}{|c|c|c|c|c|}
\hline $\begin{array}{l}\text { radical structure } \\
\text { (monomer,addition,tacticity) }\end{array}$ & $\begin{array}{c}\text { E for peroxy radical } \\
\text { (hartree) }\end{array}$ & $\begin{array}{c}E \text { for allyl radical } \\
\text { (hartree) }\end{array}$ & $\begin{array}{c}\mathrm{BDE}^{\mathrm{a}} \\
\text { (hartree) }\end{array}$ & $\begin{array}{c}\mathrm{BDE}^{\mathrm{a}} \\
(\mathrm{kcal} / \mathrm{mol})\end{array}$ \\
\hline $1-5,4$ & -842.609870 & -692.227234 & 0.021822 & 13.693 \\
\hline $1-5,2$ & -842.599915 & -692.227234 & 0.011867 & 7.446 \\
\hline $2-5,4$ & -803.290644 & -652.905311 & 0.024519 & 15.385 \\
\hline $2-5,2$ & -803.279067 & -652.905311 & 0.012942 & 8.121 \\
\hline $3-5,4-S, S$ & -763.965593 & -613.580832 & 0.023947 & 15.026 \\
\hline $3-5,4-S, R$ & -763.965825 & -613.580832 & 0.024179 & 15.172 \\
\hline $3-5,2-S, S$ & -763.956698 & -613.580832 & 0.015052 & 9.445 \\
\hline $3-5,2-S, R$ & -763.953375 & -613.580832 & 0.011729 & 7.359 \\
\hline $4-2,3-S, S$ & -575.351100 & -424.961589 & 0.032697 & 20.517 \\
\hline 4-2,3-S,R & -575.354516 & -424.961589 & 0.032113 & 20.151 \\
\hline $4-2,5-S, S$ & -575.354508 & -424.961589 & 0.032105 & 20.146 \\
\hline $4-2,5-S, R$ & -575.354876 & -424.961589 & 0.032473 & 20.377 \\
\hline
\end{tabular}

a $\mathrm{BDE}=[E($ allyl radical $)+E($ oxygen $)]-E$ (peroxy radical). The calculated $E$ (oxygen) value is -150.360814 (hartree) 
(a)



Figure S-1. (a) NMR spectra of P-1 and (b) after heating at $100^{\circ} \mathrm{C}$ for $1 \mathrm{~h}$. 


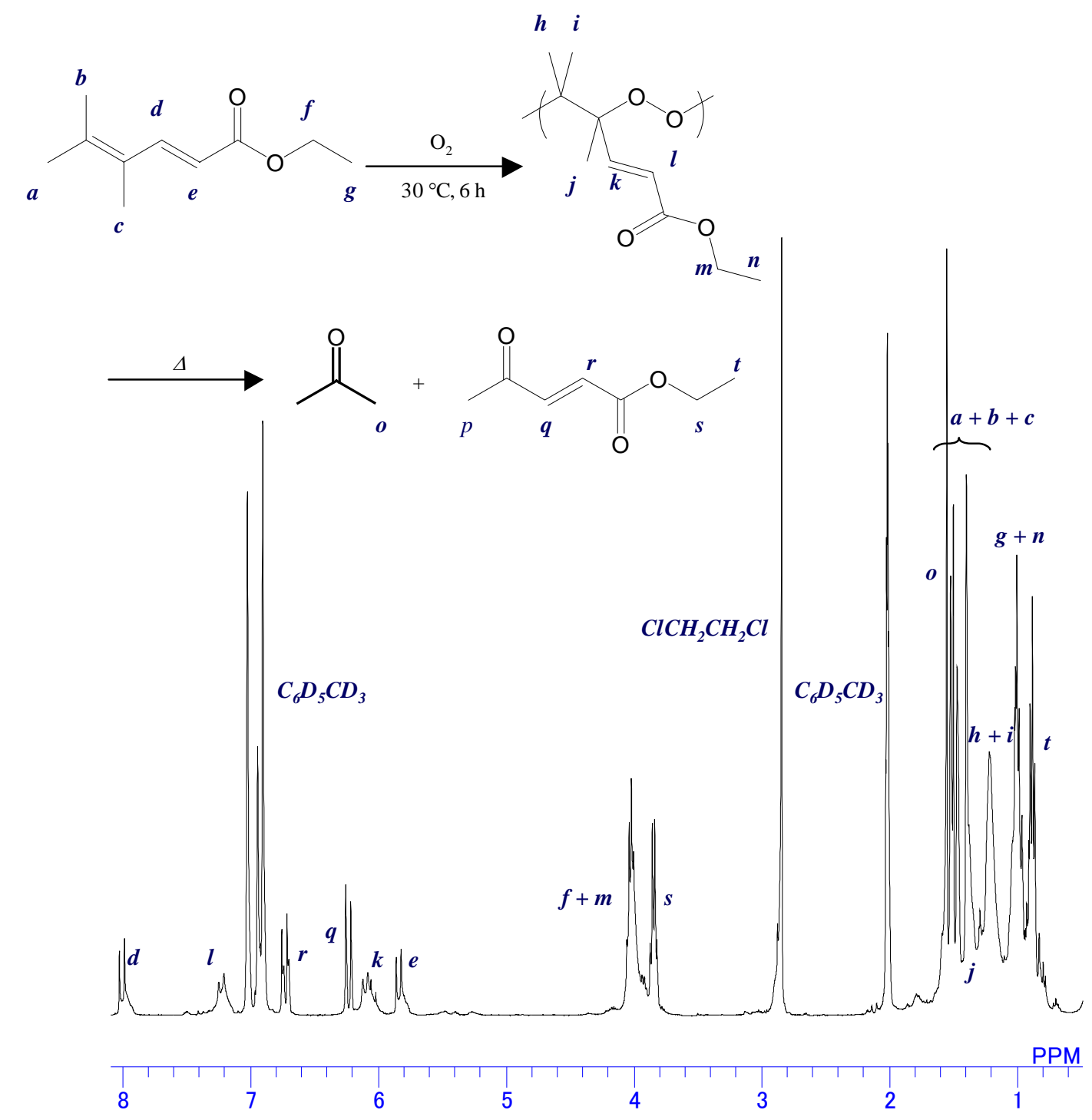

Figure S-2. NMR spectrum of the reaction mixture of 1 in toluene- $d_{8}$. Reaction conditions, $148.2 \mathrm{mg}(2.87 \mathrm{x}$ $10^{-4} \mathrm{~mol}$ ), $\mathrm{C}_{6} \mathrm{D}_{5} \mathrm{CD}_{3} 58.5 \mathrm{mg}$, 1,2-dichloroethane (internal standard) $16.0 \mathrm{mg}$, AMVN $1.0 \mathrm{mg}$, reaction temperature $30^{\circ} \mathrm{C}$, reaction time $6 \mathrm{~h}$. Monomer conversion $45.5 \%$. The ratio of degradation products to the produced P-1 was approximately 1 to 1 . 
(a)

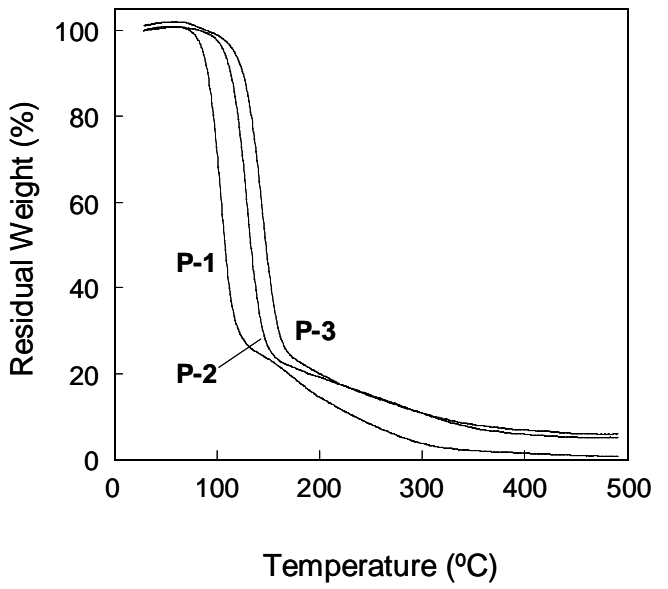

(b)



Figure S-3. (a) TG and (b) DTA curves of peroxide polymers P-1, P-2, and P-3.

Table S-2. Summary of Preparation and Thermal Properties of Peroxide Polymers

\begin{tabular}{|c|c|c|c|c|c|c|c|}
\hline \multirow{2}{*}{$\begin{array}{l}\text { Poly- } \\
\text { peroxide }\end{array}$} & \multicolumn{3}{|c|}{ Preparation ${ }^{a}$} & \multicolumn{2}{|c|}{$\mathrm{TG}^{\mathrm{b}}$} & \multicolumn{2}{|c|}{$\mathrm{DTA}^{b}$} \\
\hline & $\begin{array}{l}\text { Yield } \\
(\%)\end{array}$ & $\begin{array}{l}M_{n} \\
\times 10^{-3}\end{array}$ & $M_{\mathrm{w}} / M_{\mathrm{n}}$ & $\begin{array}{l}T_{95} \\
\left({ }^{\circ} \mathrm{C}\right)\end{array}$ & $\begin{array}{l}T_{50} \\
\left({ }^{\circ} \mathrm{C}\right)\end{array}$ & $\begin{array}{l}T_{\text {init }} \\
\left({ }^{\circ} \mathrm{C}\right)\end{array}$ & $\begin{array}{l}T_{\max } \\
\left({ }^{\circ} \mathrm{C}\right)\end{array}$ \\
\hline P-1 & 13.4 & 5.6 & 1.4 & 86.6 & 107.9 & 68.7 & 99.6 \\
\hline P-2 & 28.9 & 4.4 & 1.5 & 104.8 & 132.2 & 82.3 & 128.6 \\
\hline P-3 & 49.3 & 3.9 & 1.6 & 119.5 & 149.8 & 107.9 & 147.5 \\
\hline
\end{tabular}

a Polymerization conditions: With bubbling oxygen for $6 \mathrm{~h}$. Monomer/solvent $=1 / 1$ in weight. Monomer/AMVN $=50 / 1$ in weight. The number-average molecular weight $\left(M_{n}\right)$ and polydispersity $\left(M_{w} / M_{n}\right)$ were determined by GPC calibrated with standard polystyrenes. ${ }^{b}$ Heating rate at $10{ }^{\circ} \mathrm{C} / \mathrm{min}$ in a nitrogen stream. $T_{95}$ and $T_{50}$ are 95 and 50 weight $\%$ temperatures, respectively. $T_{\text {init }}$ and $T_{\max }$ were determined from a differential TG or DTA curve. 
Table S-3. Results of Isothermal Degradation of Peroxide Polymers ${ }^{a}$

\begin{tabular}{lcccc}
\hline $\begin{array}{l}\text { Poly- } \\
\text { peroxide }\end{array}$ & $\begin{array}{c}\text { Temperature } \\
\left({ }^{\circ} \mathrm{C}\right)\end{array}$ & $\begin{array}{c}\text { Time } \\
(\mathrm{h})\end{array}$ & $\begin{array}{c}\text { Conversion } \\
(\%)\end{array}$ & $\begin{array}{c}\text { Product Yield } \text { (Product Ratio) } \\
(\% / \%)\end{array}$ \\
\hline P-1 & 50 & 1 & 7.8 & $15.3 / 17.0(1 / 1.11)$ \\
& 50 & 2 & 24.1 & $28.6 / 28.9(1 / 1.01)$ \\
& 50 & 3 & 35.3 & $38.3 / 37.4(1 / 0.98)$ \\
& 50 & 5 & 53.1 & $49.4 / 51.0(1 / 1.03)$ \\
& 50 & 7 & 64.0 & $62.4 / 61.0(1 / 0.98)$ \\
& 70 & 0.5 & 53.2 & $61.7 / 47.2(1 / 0.76)$ \\
& 70 & 1 & 80.5 & $77.7 / 70.1(1 / 0.90)$ \\
& 70 & 1.5 & 90.9 & $89.4 / 82.1(1 / 0.92)$ \\
P-2 & 90 & 0.5 & -100 & $81.4 / 81.8(1 / 1.00)$ \\
& 90 & 1 & & \\
& 90 & 2 & 45.8 & $52.6 / 49.0(1 / 0.93)$ \\
& 90 & 3 & 78.7 & $76.0 / 63.4(1 / 0.83)$ \\
& 90 & 5 & 88.1 & $87.8 / 69.6(1 / 0.79)$ \\
& 110 & 0.5 & 97.8 & $83.0 / 64.8(1 / 0.78)$ \\
P-3 & & 91.1 & $85.8 / 81.6(1 / 0.95)$ \\
& 90 & 1 & & \\
& 90 & 3 & 13.9 & $11.1 / 12.1(1 / 1.09)$ \\
& 90 & 5 & 34.5 & $22.6 / 24.8(1 / 1.09)$ \\
& 110 & 0.5 & 45.5 & $33.3 / 34.3(1 / 1.03)$ \\
& 110 & 1 & 62.9 & $44.9 / 42.6(1 / 0.95)$ \\
& 110 & 2 & 76.7 & $52.7 / 50.5(1 / 0.96)$ \\
& & 90.7 & $59.2 / 57.1(1 / 0.96)$
\end{tabular}

${ }^{a}$ Degradation was carried out in toluene- $d_{8}$ or benzene- $d_{6} . \quad{ }^{b}$ Degradation products were acetone and ethyl 2-acetylacrylate for P-1, acetone and fumaraldehyde monoester for P-2, and acetoaldehyde and fumaraldehyde monoester for P-3. 